\title{
A Letter of Consequence: Referral Letters From General Practitioners to Secondary Mental Health Services
}

\author{
lan Shaw \\ Katherine M. Clegg Smith \\ Hugh Middleton Louise \\ Woodward
}

The referral letter is a key instrument in moving patients from primary to secondary care services. Consequently, the circumstances in which a referral should be made and its contents have been the subject of clinical guidelines. This article is based on a project that demonstrated that physicians do not adhere to clinical guidelines when referring patients to secondary mental health services. This research supports earlier findings into noncompliance with guidelines by general practitioners (GPS). The authors briefly note possible reasons, which have been the subject of some debate. They also present a content analysis of referral letters to demonstrate the important ways in which they differ from guideline criteria. However, their central argument is that the role of the referral letter in relation to the GP's repertoire of treatments has not been understood fully. Such understanding implies the need for a reexamination of the support available for GPS.

Keywords: referral letter; general practitioners; ritual; communication

ncreased emphasis on evidence-based practice and a growing awareness of 1 inequalities of health care delivery form a background to the increasing development of treatment protocols and guidelines. Most recently, this trend has been strengthened by explicit government policy (Department of Health, 1998). This proposes a number of top-down mechanisms for standardizing practice and reducing inequalities: the National Institute for Clinical Excellence, National Service Frameworks, the Commission for Health Improvement, and structures to support clinical governance and continuing medical education. Evidently, these mechanisms are designed to develop and monitor the use of protocols and guidelines that will reflect nationally agreed patterns of service delivery and their standards. Clinicians clearly cannot be expected to read every research study relevant to their practice as it is published.

The logic of guidelines is essentially algorithmic, that is, it guides its users to courses of (diagnostic or therapeutic) action, dependent upon a stated prior condition: "if... then" logic. The logic is also normative, that is it tells the clinician what ought to be 
done. In general, guidelines do not claim to determine clinical action completely, and degrees of discretion are left. (Harrison, 1998 p. 16, emphasis in original)

Protocols and guidelines can be seen as ways of managing demand and designed to be primarily in the interests of the services organization and/or government rather than patients (Harrison, 1998). Despite the creation of the U.K. National Health Services (NHS) Primary Care Trusts, independently contracted general practitioners remain among the main architects of primary care policy in the sense that the aggregation of their separate decisions and behaviors adds up to a patterned agency behavior overall (Lipsky, 1989). Such discretionary activity is essential to primary care because of the complex nature of the presenting clients, and his or her situation needs to be weighed when considering a course of action. Clinical guidelines are one of the tools intended to change practitioner behavior and to standardize levels of care. They would also serve to bend discretionary activity toward the preferences of the bureaucratic center:

Executives can affect policy even though they cannot intrude into the discretionary activity itself. They can do this by attempting to increase the possibility that outcomes on the whole will be more favorable to the preferred policy direction. (Lipsky, 1980, p. 15)

However, there is strong evidence that clinical guidelines do not invariably provide the "successful outcome" aimed at by managers of service organization (Kendrick, 2000). Previous studies indicate that the processes of formulation and implementation of protocols play as important a part in determining whether guidelines are likely to be effective as their content (Department of Health, 1984; Feder et al., 1995; Grimshaw \& Russell, 1994; Haines \& Feder, 1992). A key factor is the extent to which eventual users of the guidelines have been involved in their development. Haines and Feder presented their position succinctly: "Conflicts may arise when guidelines dreamed up by specialists prove unworkable in primary care" (p. 785). Delamothe (1993) went even further, suggesting that clinical guidelines have the best chance of success when the people who are going to use them draw them up. Unless care is taken to respect these issues, there is a risk that government policies will be frustrated by predictable resistances to change.

If guidelines and other attempts to alter practice are to be effective, they have to give very careful consideration to the beliefs, attitudes, norms, and impinging circumstances of practitioners expected to use them (Lawton \& Parker, 1999).

One area of practice in which these issues are particularly pertinent is the referral from primary to secondary care services of patients considered to have mental health problems. Up until approximately 2 decades ago, general practitioners struggled to recognize or acknowledge treatable anxiety and depression in many patients presenting to them (Freeman, Gillam, Shearin, \& Plamping, 1997). Since then, however, there has been a proactive approach, such as guidelines, in identifying and managing such illnesses. Despite this, there remains a long history of perceived disparities between the way mental illness is construed by primary care practitioners and the way psychiatrists construe it. As a result, disparities exist in the way these two groups perceive referrals. Many specialized service providers frequently use the expression "inappropriate referral." On the other hand, there are 
not infrequent instances of patients who might benefit from specialized services and yet are deprived of those benefits because they are not referred.

A challenge for GPs, and for health services in general, is identification of their patients' mental health needs and the provision of effective and appropriate management. GP referral rates for mental health-related illnesses are said to be variable (Burbach \& Harding, 1997; Creed, Gowrisunkur, Russell, \& Kincey, 1990; Cummins, Jarman, \& White, 1981; McWhinney, 1969; Robertson, 1970), and the disparity between what is seen as an appropriate referral by GPs and by psychiatric services continues, within the United Kingdom, to remain unclear and inconsistent. Burbach and Harding claimed, "The 'referral threshold' on which the GP decides to refer, appears to be unique to each individual GP and is influenced by a complex interplay of factors" (p. 67). Although this might reflect in part varying attitudes and preferences toward the management of patients with mental health problems, there still remain disparities between referral patterns. Findings from research suggest that those GPs who write detailed referral letters refer more often to psychologists and less often to psychiatrists compared to those who write poor referral letters (Creed et al., 1990). Furthermore, they claimed that "GPs interested in psychological treatments refer relatively infrequently in preference' of treating more patients themselves" (Ross \& Hardy, 1999). This finding would suggest that the referral process for mental health-related illness is a complex one. The concern is that there appears to be wide variation in the services offered to individuals with minor to more serious mental health problems due to the inconstancies and preferences of detection and appropriate management of the illness. Consequently, some will receive treatment that is seen to be inappropriate but from which others might benefit. The challenge is how to reconcile such difficulties and disparities.

A number of researchers have attempted to address such inconsistencies through various initiatives. Of particular interest is the Hampshire Depression Project (Stevens, Kinmonth, Peveler, \& Thompson, 1997). This was an educational program about the recognition and management of depression in primary care. Findings were that despite an educational program that was well received by participants and rated above average by experts, there were no significant improvements in GPs' ability to recognize depression or improve outcome (Thompson et al., 2000). Another initiative is the Department of Health-funded project titled Evaluating a Guidelines Outcome (EGO). This is a cluster randomized trial of the effects of guidelines on the appropriateness of referral and its correlates on consecutive referrals from 30 practices in Bristol. The guidelines in question are a local adaptation of the World Health Organization's (1996) Diagnostic and Management Guidelines for Mental Disorders in Primary Care. Early indications suggest that this intervention, too, has had little impact on practice (T. Croudace, personal communication, December 15,2002). On international comparison, similar problems in the detection and management of depression are to be found in countries such as the United States and the Netherlands. "Research has demonstrated that even when a diagnosis of depression is made the treatment of the illness is often varied" (Gonzales, Magruder, \& Keith, 1994, p. 253) between psychotropic medication and psychiatric intervention. It has subsequently been argued that doctors are more likely to be influenced in their practice by their and their colleagues' experience with similar types of patient than by guidelines (Harrison, 1998). This is highly consonant with the individualistic ethic of the practice of medicine. 
To investigate these matters further, we have reviewed the contents of general practitioners' letters of referral to psychiatrists and community mental health teams. Only about 1 in 10 patients presenting in primary care with psychological problems are referred for specialized management. The determinants of that choice are obscure and difficult to define (Goldberg \& Huxley, 1980). Referral behavior varies across both professions and countries. In areas of the United States, for example, referrals made to urban psychiatrists by rural GPs are avoided whenever possible. This was seen to be a result of two specific hindrances: "the lack of available services and long waiting times" (Geller, 1999, p. 330). Such GPs rely heavily on mental health counselors for management of patients with depression. Despite such variation, the referral letter represents a nodal point in the decision-making process. Although frequently very brief, these letters encapsulate the referrer's reasons for making the request and, not infrequently, his or her expectations of its outcome. Furthermore, it could be argued that if referral guidelines were influencing referral practices, then reference would be made to them in such referral letters. From a public health perspective, should such referral letters reflect appropriate decisions in the management of patients' conditions, then the recompense is a reduction in the huge economic burden of "minor" psychiatric morbidity in terms of costly interventions and referrals to psychiatric services (Freeman et al., 1997). Furthermore, it would reflect more salient treatment for the individual.

Thus, we have carried out a two-stage study. First, we compared referral letters from practices that were exposed to referral guidelines with those from practices that were not. Subsequently, we investigated the wording of both sets of referral letters, seeking themes that might reflect the grounds on which the decision to refer is made in practice.

\section{METHOD}

\section{Developing Clinical Guidelines}

A set of guidelines was developed by adapting those of the World Health Organization (1996). The resulting document covered 16 areas of psychiatric or psychological disability, and information was given about characteristic presenting complaints, diagnostic features, differential diagnosis, and management guidelines for each condition. The management guidelines included

- recommendations about the provision of information to the patient and his or her family,

- specific counseling to the patient and his or her family,

- the use of medication, and

- the criteria for specialist consultation.

The document was initially developed through consultation between consultant psychiatrists, senior nurses, and clinical psychologists providing a service to a semirural .population of some 300,000 . One of the consultant psychiatrists and a community nurse manager then presented an initial draft to a small number of general practitioners serving that area at a series of practice meetings. We obtained practitioners' feedback at a subsequent series of practice meetings, and we collated 
it and incorporated it into a final version of the document. The final version can therefore be regarded as a consensus statement based on the work of the WHO, but also one that takes into account the local views of stakeholding general practitioners, clinical psychologists, psychiatric nurses, and psychiatrists. The guidelines included indications of the information to be included in referral letters.

\section{Source of Letters}

The investigation was carried out in an area served by a sectorized psychiatric service. Two community mental health teams are based in each of two areas of declining mining and light industrial activity. These teams are each supported by two consultant psychiatrists. There was a third community mental health team based in an area dominated by agriculture and declining pit villages, and a fourth serving a medium-sized market town and its surrounding rural environments. Thus, the sociodemographic characteristics of the area vary. Similarly, the characteristics of primary care practices serving the area vary widely, from large group practices with 10 or more partners serving a population of up to 20,000 to a partnership of 2 part-time practitioners serving a population of 1,600. On the whole, the more affluent areas tend to be served by a smaller number of larger practices, whereas the less affluent areas are served by a larger number of smaller practices.

Ten GP practices were chosen for inclusion in this research. We obtained consent to use the GPs' referral letters as a data source before subjecting the letters to analysis. Also before analysis, we asked the GPs to confirm that they were using the relevant referral guidelines over the period examined rather than others available to general practice (e.g., Defeat Depression Campaign Guidelines). The intention was to include practices from across the sociodemographic spectrum. We matched the 10 practices according to list size and catchment area. We gave one practice from each pair a complete set of guidelines and told clinicians that these reflected the expectations of the secondary provider unit. None of the practices was informed of the intention to monitor their subsequent referral behavior. We collected the referral letters from each of these 10 practices to psychiatrists and directly to the community mental health teams over 6 months.

Each letter was photocopied at the point of entry into the service and sent to a central point. Here, the identity of the referring practice and the patient were removed, and an identifier for the referring practice was added. At the end of 6 months, this process had accumulated 198 consecutive referral letters from the 10 practices. Of these, 50 letters were randomly selected from the "guideline GPs" for analysis and matched with the same number from the control group of GPs.

\section{EVIDENCE OF THE USE OF GUIDELINES}

There were very few differences in the frequency with which designated "guidelines" expressions occurred in letters from the two groups of practices. None of the topics identified in the guidelines was found in all of the letters. Presenting complaints occurred most frequently, with 36 guideline letters referring to it. GP diagnosis (35) and medication given in primary care (34) were also relatively prevalent. The topics that occurred frequently in the guideline group were also prevalent in 
the control letters. Presenting complaints was recorded in 30 of the control group letters, GP diagnosis in 22, and medication given in 28.

There were, however, three topics that indicated possible differences between the guideline practice and the control practice letters. Previous contact with psychiatric services was recorded in 30 letters from the guideline group but only 13 from the control. This was the only expression that was mentioned with a significantly higher probability in one group of letters compared to the other, for which there was a significant difference between the two groups of letters.

Only 12 letters suggested a specific psychiatric treatment, 9 from the guideline and 3 from the nonguideline practices. In addition, 6 of the 7 letters that made any record of counseling came from the guideline GPs. This low incidence suggests that this is not a particularly prevalent practice, that it is not felt to be significant for the purposes of referral, or that access to counseling services is difficult. However, the project did not have the resources to explore that issue further.

The length of the referral letters varied from a paragraph to two pages, with no discernible difference in length between the guideline and nonguideline groups.

\section{THE SIGNIFICANCE OF REFERRAL LETTERS}

The discovery that these guidelines had little effect on the content of referral letters and, by inference, on clinical practice is in line with much previous research (Haines \& Feder, 1992; Upton, Evans, Goldberg, \& Sharp, 1999). However, the analysis of the letters and the sociological literature indicate why GPs might not comply with guidelines when writing referral letters. The guidelines provide an ideal statement of the information secondary services would like to receive. However, this might not be uppermost in the GP's mind when writing a referral letter. The distinction between generalist and specialist practitioners can best be understood with reference to the work of Fox (1980).

In the context of the referral letters, the psychiatrist or other secondary service provider is regarded as the expert, whose knowledge is restricted to a limited field, but therein it is sometimes clear and distinct. The GP, by contrast, has a good working knowledge of many fields (which are not necessarily consistent with one another). The GP's knowledge could be argued to consist of reasoned recipes of action indicating how to bring forth typical results in typical situations by typical means. These recipes indicate procedures that they trust, even though the reasons for them might not be fully understood. By following the recipes as if they were ritual (e.g., antidepressants and counseling for melancholia) the GP can obtain the desired result in the majority of circumstances without necessarily questioning the procedural steps. This knowledge of each field is usually sufficient for the practical purposes at hand. Such recipes for action are of course formalized in clinical guidelines.

As a consequence, a referral might occur because of the failure of tried and tested routines (or the referral letter might itself represent another routine). A referral letter might, therefore, be an indication not of uncertainty per se but, rather, indeterminacy in the professional practice (i.e., what that general practitioner should to do next). This might also explain why the referral letters rarely requested specific treatment. 
Parsons (1951) first argued that the technical content of a professional's function is the primary factor in determining his or her status. Becker (1961) argued the importance of medical responsibility and clinical experience as the archetypal feature of medical practice:

\footnotetext{
Medical responsibility is responsibility for the patient's well being, and the exercise of medical responsibility is seen as the basic and key action of the practicing physician.

The physician is most a physician when he exercises this responsibility, (p. 224)
}

As Parsons (1970) pointed out, this responsibility is personal and direct, and belongs to the physician who is working directly with the patient. It is also consequential, in that it requires the physician to take the blame for bad results (p. 165). This insight provides another key to understanding the significance of referral letters. A referral letter simultaneously signifies that the GP is, for whatever reason, unable to treat the patient him- or herself and that he or she wishes to pass on the responsibility for the treatment to another. Friedson (1970), in his discussion of the clinical mentality, highlighted that physicians are particularly sensitive to criticism, particularly by other doctors (p. 178). In this sense, referral letters could be construed as an indication of medical failure. This might also explain why GPs seemed reluctant to "nail their colors to the mast" by suggesting a diagnosis.

This consideration is reflected in the content of the referral letters. In the referral letters, GPs are attempting to provide an account that warrants the GP's failure to treat the patient successfully. It also reflects an appeal for assistance in ways that would not be seen by specialist practitioners as asking for special treatment. In other words, the contents of referral letters, as well as containing patient information, are concerned with legitimating the GP's request for assistance.

\section{EVIDENCE OF LEGITIMIZATION}

Although only one letter provided a life story for the person referred, 72 made some reference to factors outside of the GP's control that were contributing to the patient's difficulty. One theme that emerged from this analysis of referral letters to mental health services seemed to be that of transferring the blame onto the patient for the failure of GP's medical treatment. Patients' poor motivation or other moral characteristic was one of the themes that emerged in 14 of the referral letters. The following comments might well reflect the personalities of the patients, but the point here is that the GP felt unable to manage those personalities in primary care and were consequently referring the "problem patient" to secondary services:

She has a poor appetite and does not look after herself well.

I think it might be useful for him to go through matters with you in the sense of motivation and encouragement.

"X" rarely complies with treatment.

[Patient] has a problem of minor self inflicted harm which she describes as a means of expressing her anger and frustration.

This patient lies. 
Another theme that emerged of factors, external to the GP's control, affecting treatment can be described as psychosocial factors:

She has depression and anxiety stemming from her marriage.

She has family problems including an abusive son.

"X" lives with someone who has a personality disorder.

She would like to have more help coping with her bereavement.

By demonstrating that the patient is someone who is undergoing significant life difficulties, the GP is implying that this continuing problem explains why his or her treatment has not succeeded.

Another theme that emerged was that the referral was justified at this point for this particular patient. This theme was based on statements

- of an established history of psychiatric treatment; there was evidence that previous involvement by secondary mental health services was in itself seen as a justification for re-referral- "You saw him before in August 1997 and I wonder if you could see him again";

- that previous treatment had been helpful or had failed to come about for some reason-"[Client] has benefited from your service in the past"; or

- that there was a perceived risk of suicide or deliberate self-harm-"[Client] has expressed suicidal ideas and has made such attempts in the past"-or where the patient may be a danger to others.

A further theme that emerged was one of expressing the patient's preferences: " ' $\mathrm{X}$ ' feels that when she has had counseling on previous occasions this has been very helpful."

Only 28 of the letters made requests for one or more specific forms of treatment, though often expressed in a tentative form: "I wonder whether ECT or Lithium might be worth trying on him?" The remainder made unspecified requests for expert assessment, expert help, or an unspecified intervention:

I would value your opinion.

I look forward to your help and advice.

Your management of this patient would be highly appreciated.

There was no significant difference in the instances of mention of treatment or the detail of that treatment between the letters of the guideline and the nonguideline groups.

\section{OTHER ISSUES EMERGING}

It is possible to attribute the similarity in the frequency with which guideline expressions are used in letters from the two groups of practices to shortcomings in the process of development and dissemination of the guidelines. Although local consultation was part of the developmental process in this study, it was not as extensive or as thorough as has been used in others ( $\mathrm{T}$. Croudace, personal communication, December 15,2002). Furthermore, for financial reasons, the investment in education 
and dissemination was not as great as it has been in other studies (Stevens et al., 1997). Clearly, this is an area of medical practice in which a greater investment in consultation and training is likely, up to a point, to be rewarded by greater changes in practice. The purpose of this element of our study was to reestablish the fact that simply developing and disseminating structured guidelines is not enough in itself to alter the clinical practice significantly.

In addition, this review of the letters demonstrated a wide variation in the frequency with which different guidelines expressions occurred in both sets of letters. More than two thirds of the letters from both groups of practices referred to presenting complaints, and a similar proportion referred to the general practitioner's diagnosis, but a much smaller proportion referred to diagnostic features and/or treatment given in primary care. A surprisingly small proportion of letters from both groups of practices referred to information given to the patient or the family, or counseling given to the patient or family, as requested in the guidelines. In contrast, a large proportion of the letters from both groups of practices referred to medication that the patient was already receiving. Only a small proportion referred to specific psychiatric treatment.

Information about the patient clearly emerged as an important element of the letters' content. This was evident to a similar degree in the letters from both guideline and nonguideline groups. The nature of such information is illuminating. GPs spent much of the letters giving details about the patient as a person rather than the condition for which they were making the referral. The details of the patient's life or social condition appeared to be prioritized over more "medical" information, such as symptoms or diagnoses. The GP appeared to be making the choice to present the patient as a "distressed" individual, often understandably unable to cope with dire social circumstances, rather than a diagnostic entity or a "case" of mental illness. Previous research has tended simply to classify such personal information as inappropriate, as it is not in line with secondary doctors' expectations and desires in relation to the letters (Williams \& Wallace, 1974). However, the argument made here is that the inclusion (and prioritization) of such information can offer an insight into the differing perception of mental health problems in general practice settings.

McWhinney (1967) observed that GPs are presented with personal narratives of health in which biological underpinnings and significant life events are interwoven. Our findings appear to support McWhinney's and further suggest that in the case of mental health consultations, such personal accounts tend to be passed on by GPs to secondary services with relatively little mediation. Further research might be profitably conducted to see if such "raw" transferal is specific to mental health referrals or whether nonmedicalized accounts are also regularly found in cases of "physical" illness.

This study has also demonstrated that it might be presumptuous to judge the "nonmedicalized" aspects of GP referral letters as demonstrations of inadequate understanding of psychiatric processes or terminology, as argued by Elwyn and Stott (1994). Marinker (1978) proposed that GPs centralize their social proximity to their patients within their claim to being a distinct specialism. "Holistic" and "socially situated" knowledge of the patient is part of the rhetoric of general practice professionalism. These letters might, therefore, be interpreted as confident demonstrations of GPs' acknowledged area of expertise. GPs are experts about patients' health in relation to their social circumstances, their family, and their background. The referral letters might prioritize such information, not because GPs 
have failed to understand that psychiatrists seek medicalized information but because they are asserting the value of their own specialized knowledge.

As already highlighted, the information about the patient being conveyed within the letters would also seem to serve as a "justification" for the referral itself. Previous research has not tended to consider any purpose of the letters beyond simply passing on information. It is, however, valuable to consider these letters as being constructed within a system constrained by extremely limited resources and that is shaped by considerable interprofessional tensions. A striking feature of practice in this area is the very small proportion of all those presenting in primary care with psychological problems actually referred to specialists. Through the letters, the GPs seek to demonstrate that each particular referral is appropriate and, therefore, that they are behaving appropriately as a gatekeeper to secondary services. Reviewing this process through the window of what can be distilled from referral letters can provide insight into this process.

It is notable that referrals are often justified by social rather than biomedical information, such as the impact that the illness was having on either the patient or his or her family, the perceived risk of suicide, and the GP's personal opinion of the patient. The letters often contained a fairly detailed history of the illness by way of justification, and an assessment by the GP of the likely success of treatment for the particular patient, the value that might accrue from their being seen by specialized services. The presence of a high frequency of expressions referring either to a recurrence of illness or to re-referral is also of interest. It emphasizes the extent to which these patients are perceived as suffering from persistent or relapsing conditions. Many of the expressions that make references to re-referral appear to reflect the view that once patients' difficulties have been construed as appropriate for management by specialized services, it is right to use a lower threshold for attempting the same again when problems recur.

The identification of a lowered threshold for referral to mental health services once a label of mental illness has been applied suggests several directions for further research. Is the effect due to a change in the GP's attitude toward the patient and appropriate treatment, or does the labeling effect actually involve the patients' perceptions as much as those of the medical professionals? Is it easier for a GP to negotiate a referral to either psychiatrist or community mental health teams once a patient has an image of him- or herself as someone for whom such treatment is appropriate? It would be interesting to examine specialists' replies to the original referral letters and how the content of these affects subsequent referral letters, for example making it more acceptable to re-refer. Alternatively, the reverse might happen where a re-referral would not occur or would need additional justification. The content of specialist replies to referral letters and their impact on future referral behavior is the subject of current research by the authors.

A striking feature was that little information was furnished about the GPs' expectations or views of treatment in secondary care settings. It was as if these were a "black box" that did something mysterious and unspecified. This lack of clarity about expected treatment seems to reflect the greatest divergence between the expectations of the specialized service and the behavior of GPs. The referral guidelines clearly emphasize that referral should be purposive: to arrange for specific services that cannot be provided in primary care. The content of the letters clearly demonstrates an avoidance of a therapeutic formulation and related explicit requests. Such calls for "expert help" or "expert opinion" can be interpreted as a means of 
engaging secondary services without having to identify what is expected or required of them. One might conclude that this is the result of limited understanding of the area on the part of the GP, but that is unlikely. It seems more likely that references to the "expert" are a reflection of ritualized practice. It is also possible that the form and structure of referral letters has become ritualized over the years and therefore might be difficult to change simply by referring the GP to referral guidelines. This would support the findings of the American Tanenbaum study (Tanenbaum, 1994) that, in the final analysis, it is the traditional model that predominates in medical decision making. Interprofessional relationships within British medicine are complex, with defined and heavily protected boundaries of expertise. These letters appear to reflect this and in doing so illustrate how these boundaries can give rise to opaque, and, therefore, potentially misleading, communications.

\section{CONCLUSION}

In summary, at least three findings arise from this investigation of referral letters from general practitioners to providers of specialized psychiatric services. The first is that those making the referrals find it more appropriate to describe patients' needs and problems in holistic, social terms rather than to describe them by the use of a more technical framework. This appears to reflect the absence of a perceived discontinuity between distress and psychiatric disorder. This finding supports recent work exploring the use of social constructs by GPs in managing depression (Rogers, May, \& Oliver, 2001). The second, and perhaps unsurprising, point that this research underlines is that the letters very clearly reflect the strength of the practitioner's sense of acting as a gateway to secondary services. However, instead of turning to a medical justification for requesting specialized involvement (as required in the clinical guidelines), this tends to be achieved by reference to the degree of suffering, disruption, or perceived urgency generated by the problems in question, showing the difficulty of treatment in primary care. This illustrates the GPs' priorities rather than the information required by the psychiatrist. Finally, there seems to be a considerable unwillingness to make a clear or explicit request for a particular form of support or treatment. There appears to be significant deference to "expert help," but anecdotal evidence suggests that this is a traditional way of writing to other practitioners rather than true deference (D. Black, personal communication, March 27,2004).

We have referred to the long history of disparities between the ways in which primary care practitioners and specialist psychiatrists construe mental illness. The phenomenon of the "inappropriate referral" is widely cited by specialized services (Shaw \& Middleton, 2001). Psychiatrists tend to assume that this is because GPs construe mental illness incorrectly and, consequently, refer patients who are not actually mentally ill or fail to refer patients who are ill because they are relatively poor at case detection (Kendrick, 2000). Such analysis might miss the point. The argument here is that the GP referral has a different task to fulfill. Patients ask for emotional help in a variety of situations. Once the repertoire of primary care treatments has been exhausted, the current arrangements are such that few alternatives are available to GPs other than to refer the patient to secondary services. Simply regarding such referrals as inappropriate provides no support to the GP or the patient. 


\section{REFERENCES}

Backer, H. S. (1961). Boys in white: Student culture in medical school. Chicago: University of Chicago Press.

Burbach, F. R., \& Harding, S. (1997). GP referral letters to a community mental health team: An analysis of the quality and quantity of information. International Journal of Health Care Quality Assurance, 30(2), 67-72.

Creed, F., Gowrisunkur, J., Russell, E., \& Kincey, J. (1990). General practitioner referral rates to district psychiatry and psychology services. British Journal of General Practice, 40, 450-454.

Cummins, R. O., Jarman, B., \& White, P. M. (1981). Do general practitioners have different "referral thresholds"? British Medical Journal, 282,1037-1039.

Delamothe, T. (1993). Wanted: Guidelines that doctors will follow [Editorial]. British Medical Journal, $307,218$.

Department of Health. (1984). Effective health care. London: HMSO.

Department of Health. (1998). A first class service. London: HMSO.

Elwyn, G. J., \& Stott, N. C. H. (1994). Avoidable referrals: Analysis of 170 consecutive referrals to secondary care. British Medical Journal, 309,576-578.

Feder, G., Griffiths. C., Highton, C., Eldridge, S., Spence, M., \& Soothgate, L. (1995). Do clinical guidelines introduced with practice based education improve care of asthmatic and diabetic patients? A randomised controlled trial in general practice. British Medical Journal, 311,1473-1478.

Fox, R. (1980). The evolution of medical uncertainty. Millbank Memorial Fund Quarterly, 58, 1-49.

Freeman, R., Gillam, S., Shearin, C., \& Plamping, D. (1997). COPC depression and anxiety intervention guide. British Medical Journal, 315,135.

Friedson, E. (1970). Profession of medicine. Chicago: University of Chicago Press.

Geller, J. M. (1999). Rural primary care providers' perceptions of their roles in the provision of mental health services: Voices from the plains. Journal of Rural Health, 15, 326-333.

Goldberg, D., \& Huxley, P. (1980). Mental illness in the community: The pathway to psychiatric care. London: Tavistock.

Gonzales, J. J., Magruder, K. M., \& Keith, S. J. (1994). Mental disorders in primary care services: An update. Public Health Report, 109(2), 251-258.

Grimshaw, J., \& Russell, I. (1994). Achieving health gain through clinical guidelines, II: Ensuring that guidelines change medical practice. Quality in Health Care, 3, 45-52.

Haines, A., \& Feder, G. (1992). Guidance on guidelines. British Medical Journal, 305, 785-786.

Harrison, S. (1998). The politics of evidence-based medicine in the United Kingdom. Policy and Politics, 26(1), 15-31.

Kendrick, A. (2000). Why can't GPs follow guidelines on depression? British Medical Journal, 320,200-201.

Lawton, R., \& Parker, D. (1999). Procedures and the professional: The case of the British NHS. Social Science \& Medicine, 48, 353-361.

Lipsky, M. (1980). Street-level bureaucracy: Dilemmas of the individual in public services. Beverly Hills, CA: Sage.

Lipsky, M. (1989, July). The paradox of managing discretionary workers in social welfare policy. Paper presented at the Seminar on the Sociology of Social Security, University of Edinburgh, United Kingdom.

McWhinney, J. (1967). The primary physician in a comprehensive health service. The Lancet, 1, 91-96.

McWhinney, J. R. (1969). The foundations of family medicine. Canadian Family Physician, 9, 13-27.

Marinker, M. (1983). Should general practice be represented in the university medical school? British Medical Journal, 286, 855-859.

Parsons, T. (1951). The social system. London: Routledge Kegan Paul.

Robertson, N. C. (1979). Variations in referral patterns to the psychiatric services by general practitioners. Psychological Medicine, 9, 355-364.

Rogers, A., May, C., \& Oliver, D. (2001). Experiencing depression, experiencing the depressed: The separate worlds of patients and doctors. Journal of Mental Health, 10(3), 317-333.

Ross, H., \& Hardy, G. (1999). GP referrals to adult psychological services: A research agenda for promoting needs-led practice through the involvement of mental health clinicians. British Journal of Medical Psychology, 72, 75-91.

Shaw, I., \& Middleton, H. (2001). Distinguishing depression in primary care. Journal of Primary Care Mental Health, 5(2), 23-27.

Stevens, L., Kinmonth, A. L., Peveler, R. C., \& Thompson, C. (1997). The Hampshire Depression Project: Development and piloting of clinical practice guidelines and education about depression in primary health care. Medical Education, 31, 375-379. 
Tanenbaum, S. J. (1994). Knowing and acting in medical practice: Research outcomes. Journal of Health Politics, Policy and Law, 19(1), 27-44. Thompson, C, Kinmonth, A. L., Stevens, L., Peveler, R. C, Stevens, A., Ostler, K. J., et al. (2000). Effects of

a clinical-practice guideline and practice-based education on detection and outcome of depression in primary care: Hampshire Depression Project randomised controlled trial. The Lancet, 355, 185191. Upton, M. W. M., Evans, M, Goldberg, D. P., \& Sharp, D. J. (1999). Evaluation of ICD-10 PHC mental

health guidelines in detecting and managing depression within primary care. British journal of Psychiatry, 275,476-482. Williams, P., \& Wallace, B. (1974). General practitioners and psychiatrists-Do they communicate? British

Medical Journal, 267, 505-507. World Health Organization. (1996). Diagnostic and management guidelines for mental disorders in primary

care. Geneva, Switzerland: Author.

lan Shaw, B.A.(Hon.), D. Phil, is a reader in health policy in the School of Sociology and Social Policy, University of'Nottingham, United Kingdom.

Katherine M. Clegg Smith, E.A. (Hon.), M.A., Ph.D., is an assistant professor in the Department of Health Policy and Management, Bloomberg School of Public Health, Johns Hopklns University, Baltimore, Maryland.

Hugh Middleton, B.A., M.D., M.R.C.P., M.R.C.Psych., is a senior lecturer in psychiatry at the University of Nottingham, United Kingdom.

Louise Woodward, B.A.(Hon.), M.Sc., is a medical sociologist and mental health research program facilitator in the Research and Development Department, Duncan Macmillan House, Nottingham, United Kingdom. 